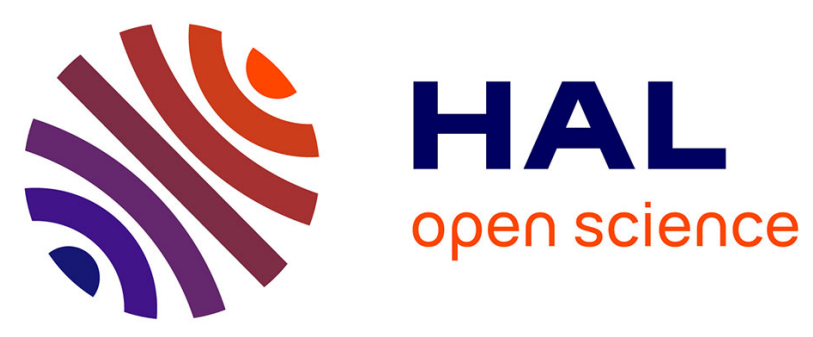

\title{
Assessing the feasibility of Nipah vaccine efficacy trials based on previous outbreaks in Bangladesh
}

\author{
Birgit Nikolay, Gabriel Ribeiro dos Santos, Marc Lipsitch, Mahmudur
}

Rahman, Stephen P. Luby, Henrik Salje, Emily S. Gurley, Simon Cauchemez

\section{To cite this version:}

Birgit Nikolay, Gabriel Ribeiro dos Santos, Marc Lipsitch, Mahmudur Rahman, Stephen P. Luby, et al. Assessing the feasibility of Nipah vaccine efficacy trials based on previous outbreaks in Bangladesh. Vaccine, 2021, pp.In Press, Corrected Proof. 10.1016/j.vaccine.2021.08.027 . pasteur-03338894

\section{HAL Id: pasteur-03338894}

\section{https://hal-pasteur.archives-ouvertes.fr/pasteur-03338894}

Submitted on 9 Sep 2021

HAL is a multi-disciplinary open access archive for the deposit and dissemination of scientific research documents, whether they are published or not. The documents may come from teaching and research institutions in France or abroad, or from public or private research centers.
L'archive ouverte pluridisciplinaire HAL, est destinée au dépôt et à la diffusion de documents scientifiques de niveau recherche, publiés ou non, émanant des établissements d'enseignement et de recherche français ou étrangers, des laboratoires publics ou privés.

\section{(1) (1) $\$$}

Distributed under a Creative Commons Attribution - NonCommercial - NoDerivatives| 4.0 
Assessing the feasibility of Nipah vaccine efficacy trials based on previous outbreaks in Bangladesh

Birgit Nikolay', Gabriel Ribeiro dos Santos², Marc Lipsitch ${ }^{3}$, Mahmudur Rahman ${ }^{4}$, Stephen P. Luby $^{5}$, Henrik Salje ${ }^{*, 2}$, Emily S. Gurley ${ }^{* 6}$, Simon Cauchemez ${ }^{* 1}$

${ }^{1}$ Mathematical Modelling of Infectious Diseases Unit, Institut Pasteur, UMR2000, CNRS, 75015 Paris, France.

${ }^{2}$ Department of Genetics, University of Cambridge, Cambridge, UK

${ }^{3}$ Harvard T.H. Chan School of Public Health, Boston, Massachusetts, USA

${ }^{4}$ Consultant

${ }^{5}$ Infectious Diseases and Geographic Medicine Division, Stanford University, Stanford, California, USA

${ }^{6}$ Department of Epidemiology, Johns Hopkins Bloomberg School of Public Health, Baltimore, Maryland, USA

${ }^{*}$ co-last authors

Corresponding author: Henrik Salje; Email: hs743@cam.ac.uk 


\section{Abstract}

Background: Nipah virus (NiV) is an emerging, bat-borne pathogen that can be transmitted from person-to-person. Vaccines are currently being developed for NiV, and studies have been funded to evaluate their safety and immunogenicity. An important unanswered question is whether it will be possible to evaluate the efficacy of vaccine candidates in phase III clinical trials in a context where spillovers from the zoonotic reservoir are infrequent and associated with small outbreaks. The objective of this study was to investigate the feasibility of conducting a phase III vaccine trial in Bangladesh, the only country regularly reporting NiV cases.

Methods: We used simulations based on previously observed NiV cases from Bangladesh, an assumed vaccine efficacy of $90 \%$ and other NiV vaccine target characteristics, to compare three vaccination study designs: (i) cluster randomized ring vaccination, (ii) cluster randomized mass vaccination, and (iii) an observational case-control study design.

Results: The simulations showed that, assuming a ramp-up period of 10 days and a mean hospitalization delay of 4 days, a cluster-randomized ring vaccination trial would require 516 years and over 163,000 vaccine doses to run a ring vaccination trial under current epidemic conditions. A cluster-randomized mass vaccination trial in the two most affected districts would take 43 years and 1.83 million vaccine doses. An observational case-control design in these two districts would require seven years and 2.5 million vaccine doses.

Discussion: Without a change in the epidemiology of NiV, ring vaccination or mass vaccination trials are unlikely to be completed within a reasonable time window. In this light, the remaining options are: (i) not conducting a phase III trial until the epidemiology of NiV changes, (ii) identifying alternative ways to licensure such as observational studies or controlled studies in animals such as in the US Food and Drug Administration's (FDA) Animal Rule.

Keywords: Nipah virus, vaccine trial, emerging pathogens 


\section{Introduction}

Vaccines can contribute to controlling the spread of emerging pathogens, but the development of such vaccines is hampered by their limited commercial value and an unclear path to licensure due to difficulties in designing trials in the context of small or unpredictable case numbers. For example, at the start of the Ebola outbreak in West Africa, several candidates were promising in animal studies but none had been given to humans because of the small size and unpredictability of prior outbreaks. This resulted in lengthy delays in being able to use the vaccine for outbreak control [1]. These difficulties were one motivation for the creation of the Coalition for Epidemic Preparedness Innovations (CEPI), with the mission to accelerate the development of vaccines through proof-of-concept, safety, and immunogenicity studies, to enable efficient efficacy testing and licencing in the case of a health emergency, and initiatives to develop vaccines for emerging infectious diseases where there was no clear pathway to a phase III clinical trial [2]. CEPI has announced financing for the development of vaccine candidates against seven emerging pathogens prioritized by the World Health Organization (WHO) including severe acute respiratory syndrome coronavirus 2, Ebola virus, Middle East respiratory syndrome coronavirus (MERS-CoV), Lassa virus, Rift Valley fever virus, chikungunya virus, and Nipah virus (NiV), as well as for capacity building to develop vaccines against an unknown disease " $X$ " [3,4].

$\mathrm{NiV}$ is an emerging, bat-borne pathogen that can also be transmitted from person-to-person $[5,6]$. While studies are currently funded to evaluate the safety and immunogenicity of four NiV vaccine candidates [4], an important question is whether, under current conditions, vaccine candidates shown to be safe and immunogenic could be tested for efficacy in phase III trials or whether alternative pathways to licensure are needed. The question arises because humans acquire NiV infections from the zoonotic reservoir infrequently and these infections are associated with outbreaks that are small and often detected late [6]. Bangladesh appears to be the most suitable place for such trials, as the epidemiology there is relatively well understood, and it is the country that reports the most NiV outbreaks [6]. However, even in Bangladesh, NiV cases remain rare: the country reports seven spillovers from the zoonotic reservoir into human populations per year on average [7]; outbreaks have never extended beyond five generations or 34 cases; and only $\sim 10 \%$ of cases transmit the virus to another person [6].

Computational simulations of disease outbreaks can inform the design of vaccine trials and have been previously used to identify the optimal trial strategy during the Ebola outbreak in West Africa and for other aspects of vaccine trial design and interpretation [8-10]. To draw valid conclusions, it is important that these simulations accurately reproduce key outbreak characteristics, which can be difficult for emerging pathogens where detailed knowledge about transmission dynamics and natural history of infection is unavailable [11]. For NiV, such information has been collected for more than 10 years of routine surveillance in Bangladesh [6] and can now be used to inform simulations that reproduce NiV transmission and allow us to test different trial designs. The aim of this study was to assess the feasibility of a phase III vaccine trial for NiV considering the current epidemiological characteristics of the pathogen. We compared three study designs: (i) cluster randomized ring vaccination, (ii) cluster randomized mass vaccination, and (iii) an observational case-control study design. 


\section{Methods}

\section{Simulated trial designs}

We simulated zoonotic cases (i.e., humans who acquired infections from the bat reservoir) and secondary human cases and three phase-three vaccine evaluation designs (Figure 1).

In the simulated ring vaccination trial, the hospitalization of any NiV case resulted in the formation of a ring, defined as contacts and contacts of contacts of the ring's index casepatient. It therefore includes all potential secondary and tertiary cases in the study. The hospitalization of an infected contact of a contact resulted in the initiation of a new ring. Rings were randomized to receive immediate or delayed ( 21 days) vaccination and the number of detected cases within a pre-specified time window was compared between the study arms to estimate the vaccine efficacy. This design is broadly similar to that of the Ebola ça Suffit! trial used to test an Ebola virus disease vaccine in Guinea in 2014-5 [12].

In the cluster-randomized mass vaccination trial design, individuals were randomized at the level of small administrative areas to receive vaccination. Two geographic scales for enrollment were considered: (i) the two districts (Faridpur and Rajbari districts, with a total population of $\sim 3$ million individuals), or (ii) the five districts (additionally including Naogaon, Rangpur, and Gopalganj districts, with a total population of $\sim 6.8$ million individuals) with highest reported detection rates of spillovers from the zoonotic reservoir into humans [7]. The vaccine efficacy was estimated by comparing the total number of cases detected by each study arm.

In the case-control study design, vaccination was offered to the entire population living in the selected districts (the same geographic areas are considered as for the cluster randomized trial design). Detected zoonotic cases in these districts were then matched to uninfected controls and the vaccination status among enrolled cases and controls was used to estimate the vaccine efficacy (1- odds ratio of vaccination among cases compared to controls) [13].

For ring vaccination and mass vaccination trials, we also investigated scenarios where people were randomly assigned to the control versus vaccine arm at an individual level.

\section{Vaccine characteristics}

Vaccine efficacy is the probability that a zoonotic or secondary case is prevented because that individual has been vaccinated. As characteristics of future Nipah vaccine candidates are unknown we based the assumptions on vaccine efficacy on the Nipah vaccine target product profile defined by the World Health Organization [WHO]; i.e. a vaccine efficacy of $90 \%$ (preferred target product profile) or $70 \%$ (minimal target product profile) [14]. We further assumed that vaccine efficacy reaches its maximum after a single dose and a ramp-up period (5 to 15 days) during which efficacy is linearly increasing and that no waning of vaccineinduced immunity occurs. We also considered vaccine post-exposure effects, i.e., prevention of symptoms and transmission if vaccination occurs within 1-5 days of exposure, as previously observed in animal studies of a vesicular stomatitis virus-based NiV vaccine candidate and in many licensed viral vaccines $[15,16]$. For population-based studies (mass vaccination trials and case control studies), we assumed that vaccination is rolled-out every 1-10 years (vaccination frequency) with a vaccination coverage of either $70 \%$ or $90 \%$. We allowed the 
vaccination coverage to vary over time depending on births of susceptible individuals and deaths of vaccinated or susceptible individuals.

\section{Simulating NiV zoonotic cases and interhuman transmission chains}

We simulated NiV zoonotic cases in Bangladesh assuming a Poisson distribution with a mean annual zoonotic spillover detection rate of 0.04 per 1 million people as reported in 2007-2018 [7]. We further simulated zoonotic cases in the two districts (average annual zoonotic spillover detection rate of 0.7 per 1 million people) or the five districts (average annual zoonotic spillover detection rate of 0.1 per 1 million people) with highest reported zoonotic spillover detection rates [7]. We accounted for population dynamics assuming predicted annual birth and death rates for Bangladesh [17].

We simulated interhuman transmission of NiV following the occurrence of a zoonotic case until the end of the transmission chain using a branching process model. For each NiV case we drew the number of secondary cases (i.e. cases infected through interhuman transmission) from a negative binomial distribution with reproduction number $\mathrm{R}=0.20$ and overdispersion parameter $\mathrm{k}=0.06$ as observed for cases reported during 2007-2014 (a time period of constant, systematic NiV surveillance) [6,7]. For each secondary NiV case, we drew the time from disease onset in the infector to infection from a discretized gamma distribution with mean 4 days and standard deviation (SD) 2 days and the time from infection to symptom onset in the secondary case from a gamma distribution with mean 10 days and SD 2 days [6]. We drew the time from disease onset to hospitalization of a case from a discretized gamma distribution with mean 5 days and SD 2 days (as reported in Bangladesh during 2001-2014 [6]) or with mean 1 day and SD 1 day (a scenario of minimal case detection delay), and assumed that case hospitalization had no impact on interhuman transmission [6].

\section{Simulating vaccination studies}

For the cluster-randomized ring vaccination trial, we simulated 50,000 rings per trial arm to estimate the intraclass correlation coefficient (ICC) assuming a ring size of 50 individuals in the baseline scenario [18]. We then estimated the required number of rings for a study powered with $80 \%$ (and a level of significance alpha=0.05) based on the cumulative number of cases occurring in each arm within a 15-36 day window following vaccination (starting at the average incubation period plus the ramp-up period and ending 21 days later), allowing the ICC to vary between study arms [18]. We estimated the study duration based on the median time to observe the required number of rings in 1,000 simulated studies. We simulated studies for up to 150 years; if the number of required rings was not reached, we estimated the duration based on the average number of rings enrolled per year in the simulations. The ICC is equal to 0 for scenarios where randomization was set at an individual level.

For the cluster randomized mass vaccination controlled trial, we simulated 50,000 clusters per trial arm for different trial durations (10-200 years) to estimate the ICC, assuming a cluster size of 100,000 individuals in the baseline scenario and that transmission events only occur within a given cluster. We quantified the required number of clusters based on the cumulative number of cases occurring in each arm [18]. We then used regression analysis to identify the trial duration that results in a total sample size corresponding to the population of selected districts (Supplementary material). 
For the case-control study, we estimated the number of zoonotic cases required to perform a case-control study powered with $80 \%$ (and a level of significance alpha $=0.05$; one-sided test) using methods implemented in the epiR package and described by Dupont [19]. As vaccination coverage varied over time, we used the observed vaccination coverage resulting in the largest sample size. We conducted a sensitivity analysis where the vaccine coverage was instead selected so that the smallest sample size was captured. In each simulation we estimated the study duration based on the median time to observe the required number of cases in 1,000 simulated studies.

All parameter values for baseline scenarios and sensitivity analyses and their sources are summarized in Table S1 in the Supplementary material. We also assessed how improvements in case detection may reduce study duration [20].

\section{Results}

\section{Cluster randomized controlled ring vaccination trial design}

Assuming a ramp-up period of 10 days and a mean hospitalization delay of 4 days, 1,807 rings per study arm need to be enrolled to run the trial for a $90 \%$ efficacious vaccine (Figure S1), which takes around 516 years and over 163,000 vaccine doses (Figure 2 A). Even in a more optimistic scenario, where NiV cases are hospitalized on average 1 day after disease onset, the study has to run approximately 317 years and requires at least 100,000 doses. There are further reductions in study duration if the vaccine has significant post-exposure effects and a shorter ramp-up period. However, even for a vaccine with 5 days post-exposure effects and only 5 days ramp-up period, the study duration remains 110 years and requires 35,000 doses (Figure $2 \mathrm{~A}$ ). In the latter scenario, the proportion of contacts whose infection is prevented increases from $7 \%$ (no post-exposure and 10 day ramp-up) to $62 \%$ (Figure S2); infections among contacts of contacts, which without post-exposure effects are preventable at a percentage close to the vaccine efficacy, are only rarely observed for NiV (Figure S2). Study duration and number of doses for a $70 \%$ efficient vaccine are shown in Figure $2 \mathrm{~B}$. Finally, we also investigated a scenario that used a standard placebo arm where individuals were not given a vaccine instead of a delayed vaccination arm. In this scenario, we estimated that obtaining vaccine efficacy estimates would require 122 years and 38,970 doses (Figure S3).

\section{Cluster randomized controlled mass vaccination trial design}

With a vaccine efficacy of $90 \%$ and a vaccination coverage of $70 \%$ in the vaccination arm, a cluster-randomized mass vaccination trial involving the total population of Faridpur and Rajbari districts (15 clusters per trial arm) requires 43 years and 1.83 million vaccine doses (Figure 2 A). To reduce the study duration to 10 years, 163 clusters per arm would need to be enrolled, representing 3.6 times the population of the two districts (Figure S5). If we extend the study area to three additional districts, the study requires 44 years and 6.03 million vaccine doses (Figure 2A). A vaccination coverage of $90 \%$ results in a study duration of 27 years and 2.03 million vaccine doses (Figure $2 \mathrm{~A}$ ). If the vaccine is $70 \%$ efficacious, study durations are substantially longer (e.g. 63 years for a vaccination coverage of $70 \%$ in Faridpur and Rajbari districts) (Figure 2B). 
For a vaccine efficacy of $90 \%$, a target vaccination coverage of $70 \%$ and using the maximum observed vaccination coverage, a case-control study with ten controls per case requires six NiV zoonotic cases (Figure S6). If implemented in Faridpur and Rajbari districts, the study takes around seven years and 2.5 million vaccine doses, compared to five years and 7.6 million vaccine doses if the study area is extended to three additional districts (Figure 2A). For a vaccination coverage of $90 \%$, nine NiV zoonotic cases need to be observed, which takes 17 years and 3.7 million vaccine doses. If the vaccine is $70 \%$ efficacious, the study requires 17 years and 2.9 million vaccine doses (70\% vaccination coverage) (Figure $2 \mathrm{~B})$. Using the minimum observed vaccination coverage yields similar results (Figure S7).

\section{Improving case detection}

It has been estimated that the true number of NiV cases in Bangladesh may be double that observed [20]. Even if the number of detected zoonotic cases doubled, a ring vaccination trial for a $90 \%$ efficacious vaccine (10 days ramp-up, no post-exposure effects) would require 258 years (Figure 3). A mass-vaccination trial in Faridpur and Rajbari districts with a vaccine coverage of $70 \%$ would require 52 years and a case control study three years (Figure 3 ).

Conducting a vaccine efficacy study within a window of 10 years, for a ring vaccination trial, would only be feasible if more than 50 times as many zoonotic cases were detected per year, which is substantially in excess of the total number of underlying zoonotic cases estimated to occur [20]. For a mass-vaccination trial in Faridpur and Rajbari districts, eight times as many zoonotic cases would need to be detected per year.

\section{Sensitivity analysis- effect of parameter assumptions on study duration}

For the ring vaccination design, increasing the size of rings has no effect on study duration (Figure S8). A longer duration of vaccine ramp-up (e.g. 327 years for 5 days vs. 1025 years for 15 days) (Figure S8) and an earlier peak in case infectivity result in longer study duration (e.g. 516 years for a mean of 4 days vs. 794 years for a mean of 2 days) (Figure S9). For the mass-vaccination design, increasing cluster sizes has no effect on study duration while a lower vaccination frequency results in slightly longer study duration (e.g. 81 years for vaccination every 5 years vs. 88 years for vaccination every 10 years) (Figure S10). For the case-control design, increasing the number of enrolled controls per case to more than ten (e.g. 6 years for 10 controls or 15 controls) and increasing the vaccination frequency has little effect on study duration (e.g. 6 years for vaccination every 5 or 10 years) (Figure S11).

\section{Individual randomization}

The use of individual rather than cluster randomization decreased the estimated number of years required from 516 years to 56 years for the ring design in the baseline scenario and from 43 years to 26 years for the mass-vaccination design. In addition, the number of required doses dropped from 162,600 to 18,200 for the ring design and from 1.83 million to 1.61 million for the mass vaccination trial design (Figure S12). We note that individual randomization led to substantial reductions in the number of doses required as well as the required duration of the trials as compared to trials that relied on cluster randomization. This benefit of individual randomization is likely to be driven by the low mean reproductive number and the highly 
overdispersed nature of onward transmission for NiV. The benefit of individual randomization, which is also difficult to implement, is likely to be lower in other disease systems that have either a higher reproductive number or there is less overdispersion.

\section{Discussion}

Our findings suggest that in Bangladesh ring vaccination or mass vaccination trials are unlikely to be completed within a reasonable time window with current epidemiologic characteristics. The ring vaccination trial design, which has been a successful strategy for the evaluation of Ebola virus vaccine candidates, is unsuitable for NiV under current conditions, due to short interhuman transmission chains. In this light the global community has the following options to consider: (i) not conducting a phase III trial until the epidemiology of NiV changes, potentially resulting again in major delays in the use of the vaccine if a larger outbreak occurs, (ii) identifying alternative ways to licensure of the vaccine not involving clinical trials in humans through either observational studies or controlled animal studies as in the US Food and Drug Administration's (FDA) Animal Rule.

An observational case-control design may represent a viable alternative strategy, which we estimate could be completed within 7 years, even with no investments to improve case detection. Case-control designs have been previously used to evaluate vaccine efficacy in the context of routine vaccination programs, as in the case of tuberculosis, meningococcus, measles and poliomyelitis vaccines [24-28]. A practical advantage of the case-control study design is that it may raise fewer ethical concerns for participants, as vaccination is not withheld from a part of the population that would have been vaccinated otherwise, and could therefore be more acceptable to communities. A case-control design may also be used to evaluate vaccine safety by including an active or passive surveillance component for adverse vaccine effects [24].

Although the level of evidence provided by a case-control design, and therefore confidence in the resulting vaccine efficacy estimates, is lower than from gold-standard cluster-randomized trials, a case-control approach offers a feasible strategy to provide both safety and health outcome data that would otherwise be unavailable and is germane to a decision on whether or not to implement vaccine. It is important to note that such observational designs can only be attempted after the vaccine is made available for mass vaccination through some regulatory process (likely short of licensure given a lack of efficacy data). One option for casecontrol designs is following mass rollout, to rely on natural inefficiencies in the vaccination process, where some individuals or communities are missed by the vaccination campaigns and can therefore act as a control group. The appropriate selection of comparable vaccinated and unvaccinated groups requires careful consideration as it is key to limiting the risk of biased vaccine efficacy estimates from other confounding variables [21]. Such biases can be minimized by considerations in the study design e.g. through matching of controls to cases in key characteristics, such as sociodemographic characteristics, or other risk factors including occupational exposure or geographic location. A "test-negative" design, where laboratory confirmed NiV patients are considered as cases and suspected NiV patients that tested negative are considered as controls, could also help limit some biases related to healthcare seeking behavior [21-23]. It has previously been estimated that around $1 \%$ of individuals with 
suspected Nipah virus infection based on symptoms are truly infected with NiV [37]. Evaluating vaccine efficacy based on an alternative outcome that cannot be causally affected by the vaccine can help testing for potential biases in the study [21]. Further analyses that specifically explore the impact of biases on vaccine efficacy estimates would help further assess the viability of this approach.

Currently, four NiV vaccine candidates are being funded by CEPI to undergo safety and immunogenicity testing with the idea that safe and immunogenic vaccines could be used and tested - in the context of any future, large outbreaks. Under current epidemiologic scenarios, simultaneous testing of more than one vaccine candidate in phase III trials would lead to substantially longer study durations. Considering the practical limitations highlighted here, it may therefore be necessary to focus efforts to evaluate efficacy of just one vaccine candidate, and criteria for deciding on which candidate to pursue would need to be developed.

The model assumptions we used to simulate zoonotic NiV cases and interhuman transmission influenced the estimated study durations. Wherever possible, our assumptions were made using data and we consistently used optimistic scenarios from the perspective of our ability to conduct a trial within a reasonable timeframe. For example, in ring vaccination trials, we assumed that hospitalization of cases had no impact on NiV transmission, which is justified as infection control measures in Bangladeshi hospitals are often limited [29]. In some settings, hospitalization may lead to increased transmission due to superspreading events as observed in two hospital based outbreaks in India [30,31]. Further, we chose an optimistic scenario where NiV cases are diagnosed immediately after hospitalization and all of their contacts are identified and vaccinated without delays. In the absence of rapid diagnostic tests for NiV, delays in case identification would be expected, also potentially only a proportion of contacts may be identified and enrolled in the study. This however only means that the estimated trial durations (already exceeding 100 years) would be even longer when taking these limitations into account.

Improving case identification could theoretically help reduce study durations, for example through enhanced surveillance for NiV cases (increasing the geographic area of surveillance and the number of hospitals or healthcare facilities in affected areas), given that in a modelling study it has been estimated that about $50 \%$ of zoonotic cases are currently missed [20]. Such studies could focus on groups at high-risk for zoonotic infections such as individuals collecting or consuming palm sap [32]. However, there are likely to be substantial logistical constraints in identifying and targeting this population. Healthcare workers may be an alternative high risk group for NiV infection; however they are currently only rarely infected in the setting of Bangladesh as family members act as main caregivers even for hospitalized NiV patients $[6,29]$. Even with perfect surveillance with all cases detected, the ring vaccination and clusterrandomized trials would remain unfeasible. Note that while the identification of asymptomatic/mild infections by serological investigations may be a viable option for some other pathogens, the incidence of such infections with NiV is estimated to be uncommon and therefore may not provide substantial additional benefit $[6,33]$.

While clinical trials are still considered as the dominant licensure pathway, a case-control study, if appropriately designed, can provide important insights on vaccine efficacy based on human data. This means however that the study will depend upon mass vaccination and require informed consent of participants and approval by local drug authorities. Mass 
vaccination as part of a vaccine trial has been previously done for the evaluation of an unlicensed cholera vaccine in Bangladesh [34]. An alternative pathway of licensing would be the use of the Animal Rule, where well designed animal studies are considered as sufficient to prove effectiveness of a vaccine [35]. Results from these animal studies can then be bridged to humans by the use of well-established correlates of immunity. This licensure pathway has been previously applied in the case of anthrax [36]. Guidance on licensing in the case where optimal clinical trials are infeasible should be established by licensing authorities and standardized, as this problem not only exists for NiV but also other emerging pathogens. 


\section{Acknowledgements}

B. N. and S. C. acknowledge the support of the Laboratory of Excellence Integrative Biology of Emerging Infectious Diseases (Grant ANR-10-LABX-62-IBEID), the National Institute of General Medical Sciences Models of Infectious Disease Agent Study Initiative, the AXA Research Fund and the INCEPTION project (PIA/ANR-16-CONV-0005).

\section{Conflict of Interest}

M.L. has received grants from Pfizer, personal fees from Merck, personal fees from BristolMeyers Squibb, personal fees from Sanofi Pasteur, unrelated to the present work.

\section{References}

[1] Fleck F, Lesher A. The race against time. Bull World Health Organ 2015;93:7-8. https://doi.org/10.2471/BLT.15.020115.

[2] Røttingen J-A, Gouglas D, Feinberg M, Plotkin S, Raghavan KV, Witty A, et al. New Vaccines against Epidemic Infectious Diseases. N Engl J Med 2017;376:610-3. https://doi.org/10.1056/NEJMp1613577.

[3] Burki T. CEPI: preparing for the worst. Lancet Infect Dis 2017;17:265-6. https://doi.org/10.1016/S1473-3099(17)30062-2.

[4] CEPI portfolio n.d. https://cepi.net/research_dev/our-portfolio/ (accessed September $27,2020)$.

[5] Luby SP, Hossain MJ, Gurley ES, Ahmed BN, Banu S, Khan SU, et al. Recurrent zoonotic transmission of Nipah virus into humans, Bangladesh, 2001-2007. Emerging Infect Dis 2009;15:1229-35. https://doi.org/10.3201/eid1508.081237.

[6] Nikolay B, Salje H, Hossain MJ, Khan AKMD, Sazzad HMS, Rahman M, et al. Transmission of Nipah Virus - 14 Years of Investigations in Bangladesh. N Engl J Med 2019;380:1804-14. https://doi.org/10.1056/NEJMoa1805376.

[7] Nikolay B, Salje H, Khan AKMD, Sazzad HMS, Satter SM, Rahman M, et al. A Framework to Monitor Changes in Transmission and Epidemiology of Emerging Pathogens: Lessons From Nipah Virus. J Infect Dis 2020;221:S363-9. https://doi.org/10.1093/infdis/jiaa074.

[8] Hitchings MDT, Grais RF, Lipsitch M. Using simulation to aid trial design: Ringvaccination trials. PLoS Negl Trop Dis 2017;11:e0005470. https://doi.org/10.1371/journal.pntd.0005470.

[9] Bellan SE, Pulliam JRC, Pearson CAB, Champredon D, Fox SJ, Skrip L, et al. Statistical power and validity of Ebola vaccine trials in Sierra Leone: a simulation study of trial design and analysis. Lancet Infect Dis 2015;15:703-10. https://doi.org/10.1016/S14733099(15)70139-8. 
[10] Camacho A, Eggo RM, Goeyvaerts N, Vandebosch A, Mogg R, Funk S, et al. Real-time dynamic modelling for the design of a cluster-randomized phase 3 Ebola vaccine trial in Sierra Leone. Vaccine 2017;35:544-51. https://doi.org/10.1016/j.vaccine.2016.12.019.

[11] Halloran ME, Auranen K, Baird S, Basta NE, Bellan SE, Brookmeyer R, et al. Simulations for designing and interpreting intervention trials in infectious diseases. BMC Med 2017;15:223. https://doi.org/10.1186/s12916-017-0985-3.

[12] Consortium E ça suffit ring vaccination trial. The ring vaccination trial: a novel cluster randomised controlled trial design to evaluate vaccine efficacy and effectiveness during outbreaks, with special reference to Ebola. BMJ 2015;351. https://doi.org/10.1136/bmj.h3740.

[13] Verani JR, Baqui AH, Broome CV, Cherian T, Cohen C, Farrar JL, et al. Case-control vaccine effectiveness studies: Data collection, analysis and reporting results. Vaccine 2017;35:3303-8. https://doi.org/10.1016/j.vaccine.2017.04.035.

[14] WHO Target Product Profile for Nipah virus Vaccine. WHO n.d. https://www.who.int/blueprint/priority-diseases/keyaction/Nipah_virus_vaccineTPP.pdf?ua=1\&ua=1 (accessed September 11, 2020).

[15] Gallagher T, Lipsitch M. Postexposure Effects of Vaccines on Infectious Diseases. Epidemiol Rev 2019;41:13-27. https://doi.org/10.1093/epirev/mxz014.

[16] DeBuysscher BL, Scott D, Thomas T, Feldmann H, Prescott J. Peri-exposure protection against Nipah virus disease using a single-dose recombinant vesicular stomatitis virus-based vaccine. NPJ Vaccines 2016;1. https://doi.org/10.1038/npjvaccines.2016.2.

[17] World Population Prospects - Population Division - United Nations n.d. https://population.un.org/wpp/ (accessed September 11, 2020).

[18] Crespi CM, Wong WK, Wu S. A new dependence parameter approach to improve the design of cluster randomized trials with binary outcomes. Clin Trials 2011;8:687-98. https://doi.org/10.1177/1740774511423851.

[19] Dupont WD. Power calculations for matched case-control studies. Biometrics 1988;44:1157-68.

[20] Hegde ST, Salje H, Sazzad HMS, Hossain MJ, Rahman M, Daszak P, et al. Using healthcare-seeking behaviour to estimate the number of Nipah outbreaks missed by hospitalbased surveillance in Bangladesh. Int J Epidemiol 2019. https://doi.org/10.1093/ije/dyz057.

[21] Lipsitch M, Jha A, Simonsen L. Observational studies and the difficult quest for causality: lessons from vaccine effectiveness and impact studies. Int J Epidemiol 2016;45:2060-74. https://doi.org/10.1093/ije/dyw124.

[22] Sullivan SG, Tchetgen Tchetgen EJ, Cowling BJ. Theoretical Basis of the Test-Negative Study Design for Assessment of Influenza Vaccine Effectiveness. Am J Epidemiol 2016;184:345-53. https://doi.org/10.1093/aje/kww064. 
[23] Chua H, Feng S, Lewnard JA, Sullivan SG, Blyth CC, Lipsitch M, et al. The Use of Testnegative Controls to Monitor Vaccine Effectiveness: A Systematic Review of Methodology. Epidemiology 2020;31:43-64. https://doi.org/10.1097/EDE.0000000000001116.

[24] Rodrigues LC, Smith PG. Use of the case-control approach in vaccine evaluation: efficacy and adverse effects. Epidemiol Rev 1999;21:56-72. https://doi.org/10.1093/oxfordjournals.epirev.a017988.

[25] Hennessey KA, lon-Nedelcu N, Craciun MD, Toma F, Wattigney W, Strebel PM. Measles epidemic in Romania, 1996-1998: assessment of vaccine effectiveness by case-control and cohort studies. Am J Epidemiol 1999;150:1250-7. https://doi.org/10.1093/oxfordjournals.aje.a009952.

[26] Deivanayagam N, Nedunchelian K, Ahamed SS, Rathnam SR. Clinical efficacy of trivalent oral poliomyelitis vaccine: a case-control study. Bull World Health Organ 1993;71:307-9.

[27] Grassly NC, Wenger J, Durrani S, Bahl S, Deshpande JM, Sutter RW, et al. Protective efficacy of a monovalent oral type 1 poliovirus vaccine: a case-control study. Lancet 2007;369:1356-62. https://doi.org/10.1016/S0140-6736(07)60531-5.

[28] Kidd S, Ouedraogo B, Kambire C, Kambou JL, McLean H, Kutty PK, et al. Measles outbreak in Burkina Faso, 2009: A case-control study to determine risk factors and estimate vaccine effectiveness. Vaccine 2012;30:5000-8. https://doi.org/10.1016/j.vaccine.2012.05.024.

[29] Islam MS, Luby SP, Sultana R, Rimi NA, Zaman RU, Uddin M, et al. Family caregivers in public tertiary care hospitals in Bangladesh: risks and opportunities for infection control. Am J Infect Control 2014;42:305-10. https://doi.org/10.1016/j.ajic.2013.09.012.

[30] Arunkumar G, Chandni R, Mourya DT, Singh SK, Sadanandan R, Sudan P, et al. Outbreak investigation of Nipah Virus Disease in Kerala, India, 2018. J Infect Dis 2018. https://doi.org/10.1093/infdis/jiy612.

[31] Chadha MS, Comer JA, Lowe L, Rota PA, Rollin PE, Bellini WJ, et al. Nipah virusassociated encephalitis outbreak, Siliguri, India. Emerging Infect Dis 2006;12:235-40. https://doi.org/10.3201/eid1202.051247.

[32] Rahman MA, Hossain MJ, Sultana S, Homaira N, Khan SU, Rahman M, et al. Date palm sap linked to Nipah virus outbreak in Bangladesh, 2008. Vector Borne Zoonotic Dis 2012;12:65-72. https://doi.org/10.1089/vbz.2011.0656.

[33] Kumar CPG, Sugunan AP, Yadav P, Kurup KK, Aarathee R, Manickam P, Bhatnagar T, Radhakrishnan C, Thomas B, Kumar A 5th, Jayasree J, Philomina B, Kumar KGS, Thulaseedharan NK, Gupta N 5th, Rajendran R, Saritha RL, Mourya DT, Gangakhedkar RR, Murhekar MV. Infections among Contacts of Patients with Nipah Virus, India. Emerg Infect Dis. 2019 May;25(5):1007-1010. doi: 10.3201/eid2505.181352. PMID: 31002050; PMCID: PMC6478200. 
[34] Qadri F, Ali M, Chowdhury F, Khan AI, Saha A, Khan IA, et al. Feasibility and effectiveness of oral cholera vaccine in an urban endemic setting in Bangladesh: a cluster randomised open-label trial. The Lancet 2015;386:1362-71. https://doi.org/10.1016/S01406736(15)61140-0.

[35] Burns DL. Licensure of vaccines using the Animal Rule. Current Opinion in Virology 2012;2:353-6. https://doi.org/10.1016/j.coviro.2012.01.004.

[36] Beasley DWC, Brasel TL, Comer JE. First vaccine approval under the FDA Animal Rule. Npj Vaccines 2016;1:1-3. https://doi.org/10.1038/npjvaccines.2016.13.

[37] Naser AM, Hossain MJ, Sazzad HM, Homaira N, Gurley ES, Podder G, Afroj S, Banu S, Rollin PE, Daszak P, Ahmed BN, Rahman M, Luby SP. Integrated clusterand case-based surveillance for detecting stage III zoonotic pathogens: an example of Nipah virus surveillance in Bangladesh. Epidemiol Infect. 2015 Jul;143(9):1922-30. doi: 10.1017/S0950268814002635. Epub 2014 Oct 24. PMID: 25342551; PMCID: PMC4456770. 
Figure 1. Vaccine trial study designs.

Figure 2. Number of years and vaccine doses required for different study designs assuming a vaccine efficacy of $90 \%(A)$ and $70 \%(B)$. Estimates are presented for a ring size of 90 individuals (ring vaccination trial), a cluster size of 100,000 individuals and a vaccination frequency of five years (mass vaccination trial and case control study), and ten controls per case (case control study). Cluster-Randomized Ring vaccination trial (CRR); Cluster-Randomized Mass-vaccination Trial (CRMv); Case control study (CC); Mean hospitalization delay (Hosp); Post-exposure duration (Postexp); Vaccine coverage (Vcov); Duration of ramp-up period (dramp). Study durations are truncated at 200 years.

Figure 3. Number of years and vaccine doses required for a $90 \%$ efficacious vaccine if the number of detected spillovers from the zoonotic reservoir into human populations doubled. Estimates are presented for a ring size of 90 individuals (ring vaccination trial), a cluster size of 100,000 individuals and a vaccination frequency of five years (cluster randomized trial and case control study), and ten controls per case (case control study). Cluster-Randomized Ring vaccination trial (CRR); Cluster-Randomized Mass-vaccination Trial (CRMv); Case control study (CC); Mean hospitalization delay (Hosp); Post-exposure duration (Postexp); Vaccine coverage (Vcov); Duration of ramp-up period (dramp). Study durations are truncated at 200 years. 


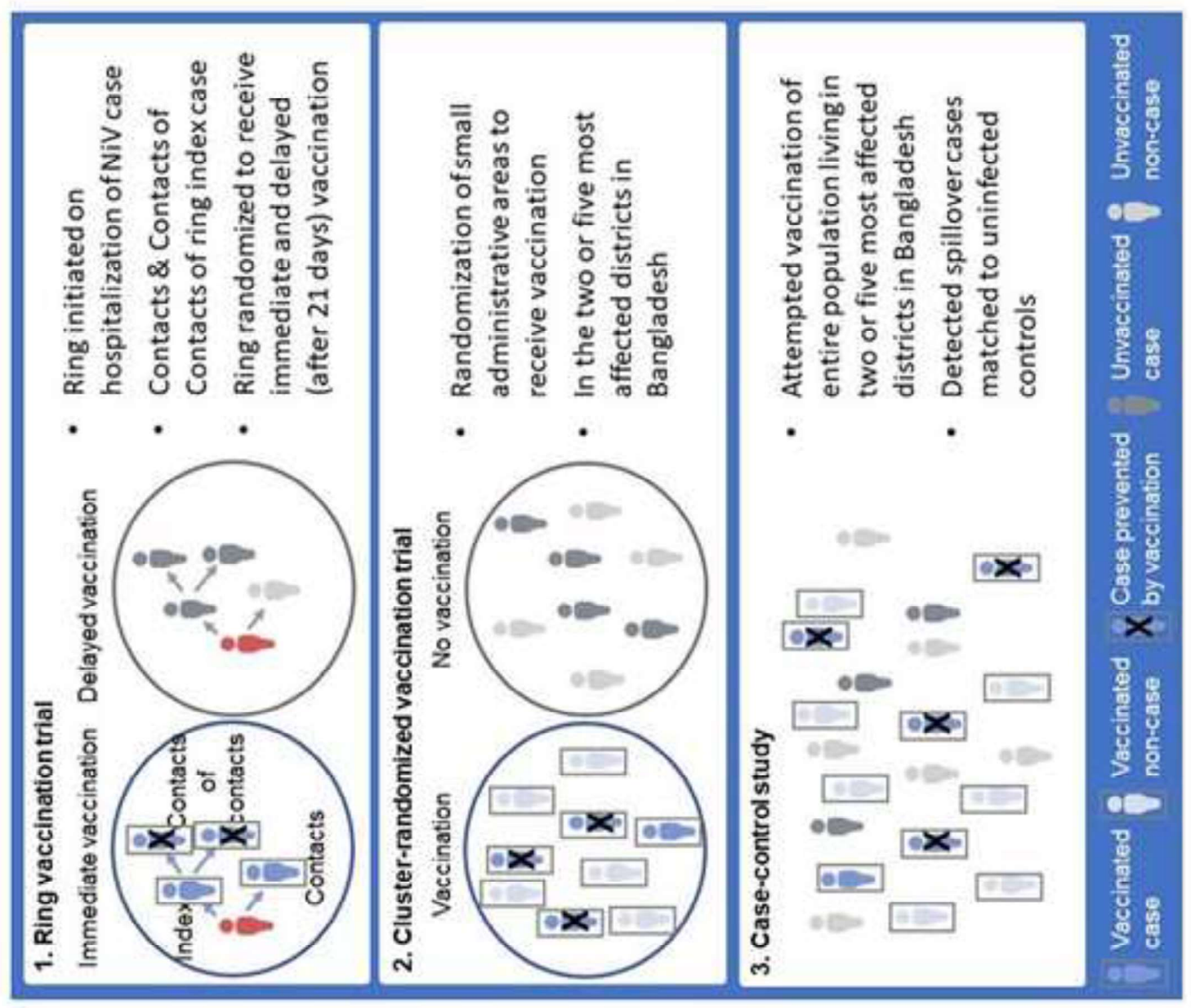


웅으유

III II i

을 을

뜐

๑்

的

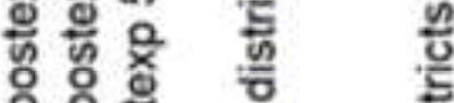

뜽

을우유. 잉

훙ㅇㅇ

눙

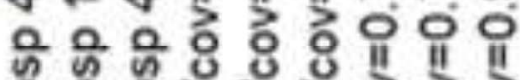

우우우>>>긍 흥

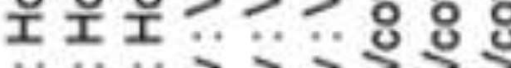

$\alpha \propto \alpha \gtreqless \geq \geq>>$

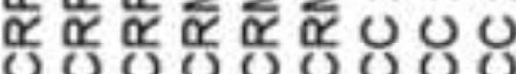

응ㅎㅇㅇㅡ

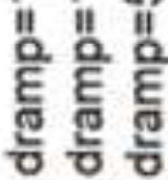

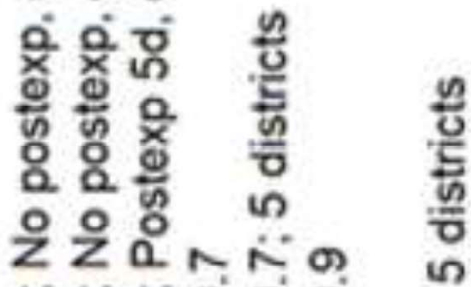

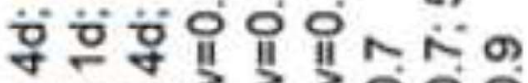

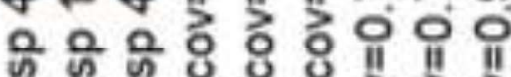

우우우고잉 항

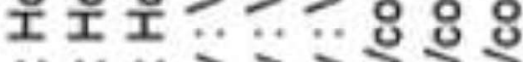

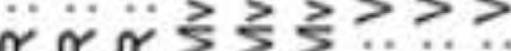

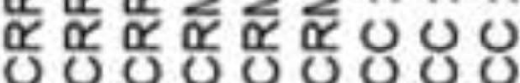
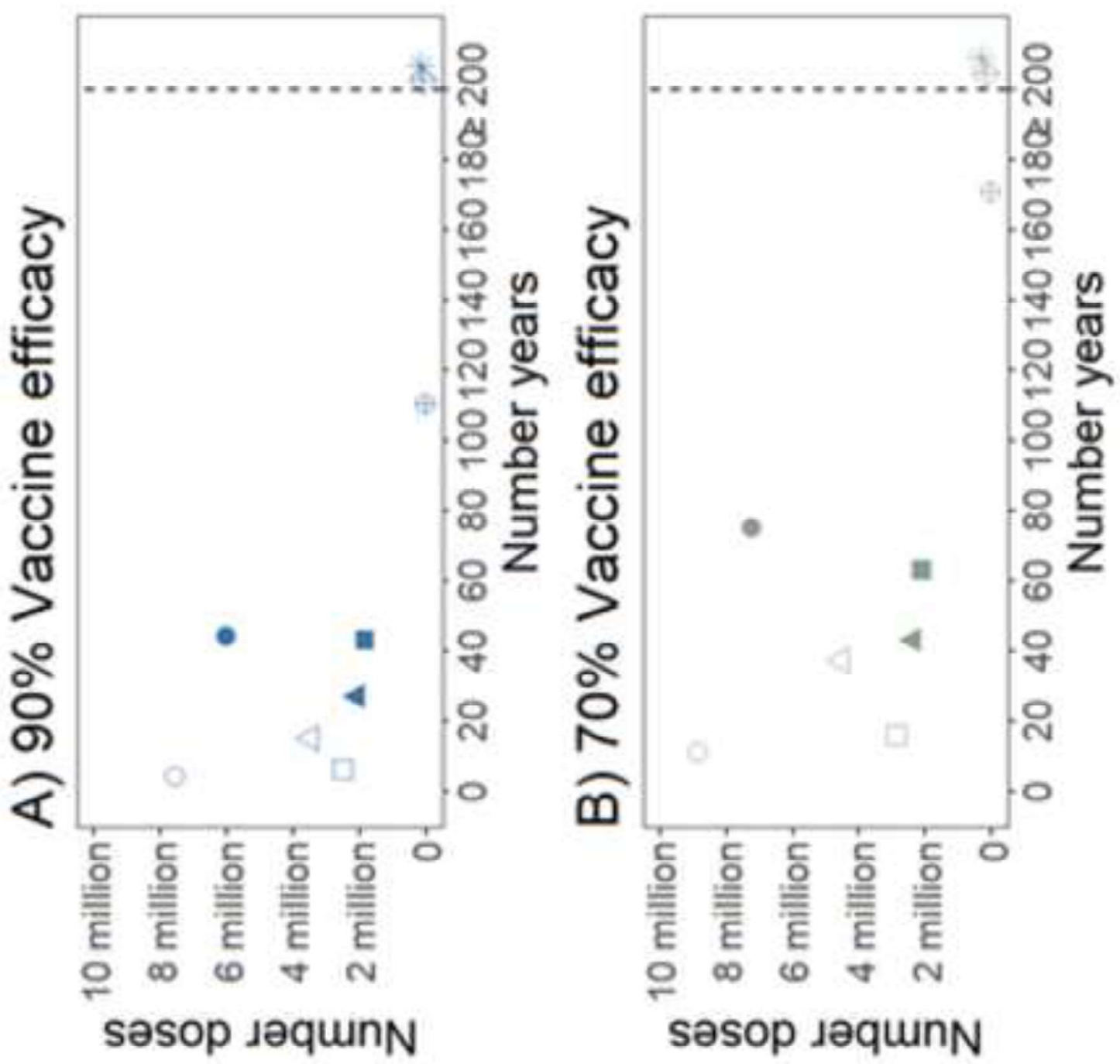

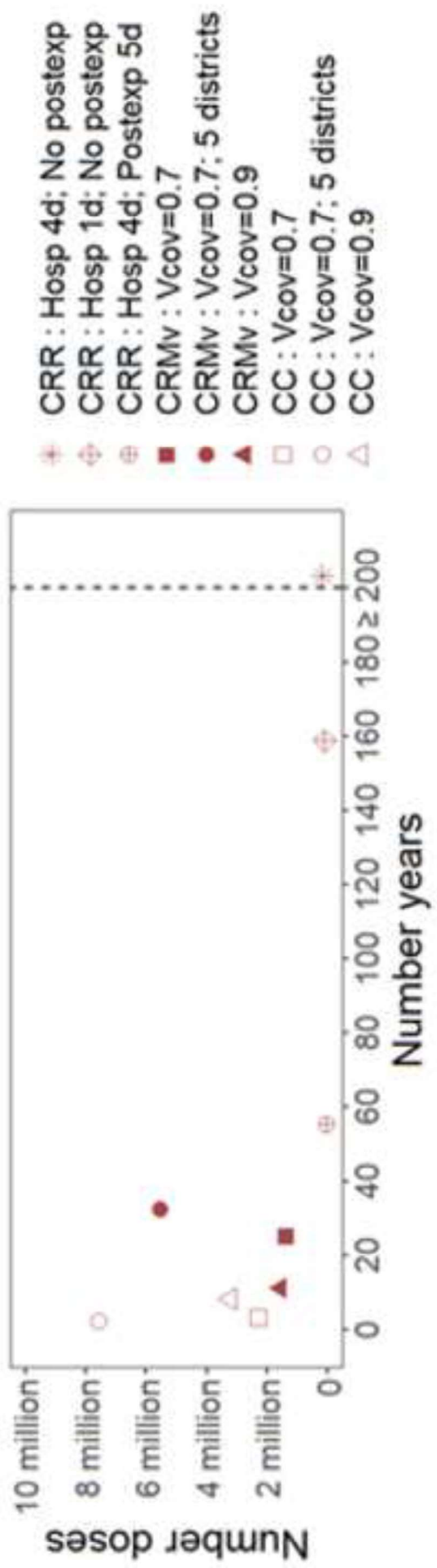
Click here to access/download Supplemental Files

NiV_vaccine_suplement_rev.docx 